\title{
Late-Onset Narcolepsy: A Case Report with Brief Review of the Literature
}

\author{
Jeny Jacob ${ }^{1}$ Rajesh Venkataram ${ }^{2}$ Nandakishore Baikunje ${ }^{2}$ Rashmi Soori ${ }^{3}$
}

\author{
${ }^{1}$ Department of General Medicine, K.S. Hegde Medical Academy, \\ Mangalore, Karnataka, India \\ 2Department of Pulmonary Medicine, K.S. Hegde Medical Academy, \\ Mangalore, Karnataka, India \\ ${ }^{3}$ Department of Anaesthesiology, K.S. Hegde Medical Academy, \\ Deralakatte, Mangalore, Karnataka, India \\ J Health Allied Sci ${ }^{N U}: 2021 ; 11: 107-109$
}

\begin{abstract}
Address for correspondence Rajesh Venkataram, MD, Department of Pulmonary Medicine, K.S. Hegde Medical Academy, Deralakatte, Mangalore, 575018 Karnataka, India (e-mail: rajdocmmc@gmail.com).
\end{abstract}
Abstract
Keywords
- narcolepsy
- excessive daytime sleepiness
- Multiple Sleep
Latency Test

Narcolepsy, a sleep disorder, has its onset in childhood and early adulthood but rarely in older adults. This case report focuses on a man in his late fifties who was noticed to have excessive daytime sleepiness during his stay in our hospital for an unrelated medical ailment. He was further evaluated with overnight polysomnography and next day multiple sleep latency test which confirmed the diagnosis of narcolepsy.

\section{Introduction}

Narcolepsy is a rare, disabling sleep disorder, with average age of onset between 20 and 30 years, with a prevalence of 20 to 30 per 100,000 population. ${ }^{1}$ Narcolepsy affects both genders, with a slight male preponderance. ${ }^{2}$ The cardinal symptoms of narcolepsy are excessive daytime sleepiness (EDS), cataplexy, hypnagogic hallucinations, and sleep paralysis. ${ }^{1}$ Multiple Sleep Latency Test (MSLT) is the confirmatory test done to diagnose narcolepsy. ${ }^{3}$ The cause of human narcolepsy remains unknown, however an autoimmune process acting on genetic and environmental background are thought to be involved. ${ }^{3}$

\section{Case Report}

A 58-year-old male was being treated for acute sinusitis in our hospital. During the hospital stay, he was noticed to have EDS. On further enquiry he gave history of sudden sleep attacks during day and vivid dream-like hallucinatory images at the beginning of sleep attacks (hypnagogic hallucinations). He revealed to be having these complaints since past 8 years. There was no history suggestive of cataplexy, loss of consciousness, loss of muscle power at the start/after the sleep attacks, vomiting, visual disturbances, and seizures.

published online

February 13, 2021
DOI https://doi.org/ 10.1055/s-0040-1722802 ISSN 2582-4287.
He was not on any prior medications and there was no history of substance abuse, head trauma, or any central nervous system illness in the past. Epworth Sleepiness Scale was administered and he had a score of 16 . His body mass index was $24.6 \mathrm{~kg} / \mathrm{m}^{2}$. General physical examination and upper respiratory tract examination was unrewarding. A thorough central nervous system examination revealed no neurological deficits. He was advised to undergo MSLT after recovery from acute sinusitis. As per the standard protocol, overnight Level 1 polysomnography (PSG) was done to rule out obstructive sleep apnea (OSA) (which is a common cause for day time sleepiness) and other sleep disorders. PSG showed apnea-hypopnea index of 2.3 hour, which is normal. Respiratory disturbance index was also 2.3/hour as the patient did not have any episodes of respiratory effort-related arousals. Additionally, out of 551 minutes of recording, he had slept for 440 minutes resulting in sleep efficiency of $80 \%$ and there was no evidence suggestive of any other sleep disorder. Next day, MSLT was done which showed mean sleep latency of 42 seconds and two sleep-onset rapid eye movement sleep periods (SOREMPs) in second and third nap opportunities (-Figs. 1 and 2). A diagnosis of narcolepsy without cataplexy was made. He was started on tablet modafinil as per the neurologist's advice. 


\section{Hypnograms}

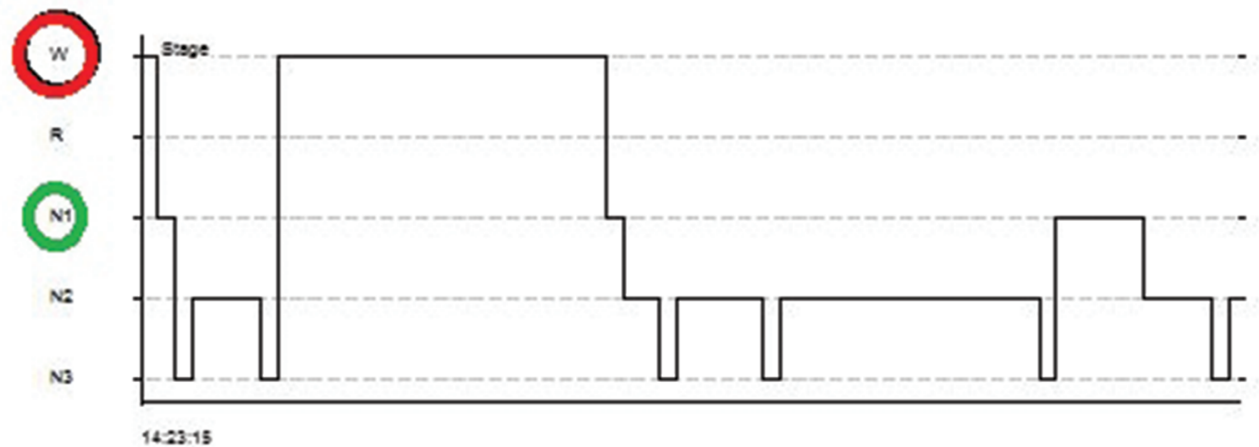

Summary

\begin{tabular}{|l|c|} 
& Acq 1 \\
\hline Lights OFF & $14: 23: 15$ \\
Sleep Onset (SO) & $14: 23: 45$ \\
Lights ON & $14: 55: 15$ \\
Time in Bed & $00: 32: 00$ \\
Tot Sleep Time & $00: 22: 00$ \\
Sleep latency & $00: 00: 30$ \\
REM latency &
\end{tabular}

Average sleep latency ( 1 values) : :00:00:30

Average REM latency ( 0 values) :/

Fig. 1 Hypnogram of third nap opportunity of Multiple Sleep Latency Test (MSLT) showing sleep latency of 30 seconds. Subject has transitioned from wake status (marked as W, encircled in red) to N1 stage of non-rapid eye movement (NREM) sleep (marked as N1, encircled in green).

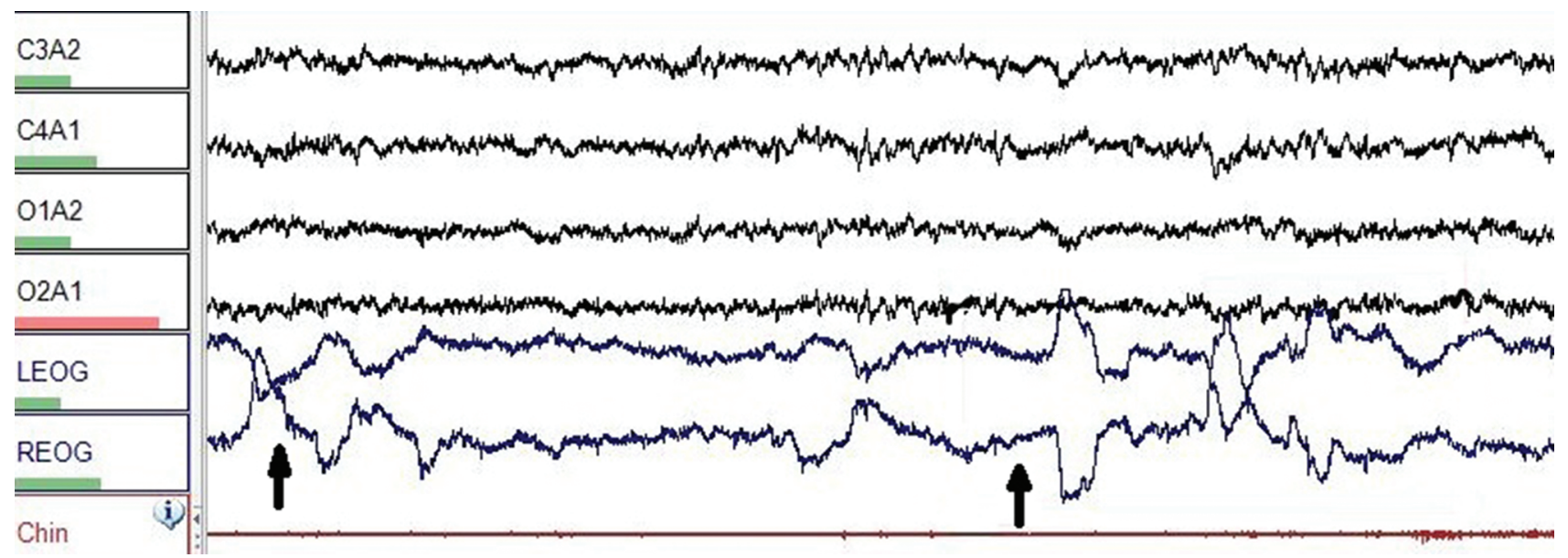

Fig. 2 Electroencephalogram showing sleep-onset rapid eye movement sleep period. Black arrow pointing toward conjugate eye movements detected in electrooculogram suggestive of rapid eye movement sleep.

\section{Review of Literature}

Narcolepsy is a sleep disorder characterized by difficulty in sustaining wakefulness. Narcolepsy typically affects children and adolescents/young adults but rarely older adults. Two peaks of onset-at ages 15 to 25 years and ages 30 to 35 years-are known. Once established, the disease persists for life. It is characterized by EDS, sleep paralysis (muscle paralysis upon awakening), hypnagogic hallucinations (vivid and sometimes frightening hallucinations at the start of sleep), hypnopompic hallucinations (hallucinations at the end of sleep), and cataplexy (sudden, episodic, emotionally triggered muscle weakness). EDS is usually the first symptom, with cataplexy and other symptoms developing over the next few months and persisting for life. ${ }^{2}$ 
There are two main types of narcolepsy:

1. Narcolepsy with cataplexy:

Itconstitutes approximately 60 to $70 \%$ of narcolepsy. ${ }^{3}$ Cataplexy are transient, typically bilateral muscle weakness commonly affecting the jaws or face rather than lower limb with consciousness fully preserved, which are most often triggered by strong emotion. The most common precipitants of cataplexy are laughter (92\%), anger (70\%), and startle reaction (55\%). Cardinal feature of cataplexy is that the muscular weakness resolves immediately after an attack. ${ }^{1}$

2. Narcolepsy without cataplexy:

Here, except for cataplexy all other symptoms are present.

\section{Etiology}

The orexin peptides (orexin A and B) produced in the hypothalamus are involved in maintaining wakefulness and regulating transitions between sleep and wake. Approximately 90\% of the orexin-producing neurons are lost in patients with narcolepsy with cataplexy. ${ }^{4,5}$

The neuropathology of narcolepsy without cataplexy is much less understood. They may be caused by less severe injury to the orexin neurons. ${ }^{6}$

Narcolepsy has strong human leukocyte antigen (HLA) association and approximately $90 \%$ of the patients with narcolepsy are found to have HLA DQB $1^{*} 0602$.

Other risk factors are intracranial tumors, neurosarcoidosis, multiple sclerosis, paraneoplastic syndromes, Neimann-Pick type C disease, head trauma, and Parkinson's disease.

\section{Diagnosis}

Simplified diagnostic criteria for narcolepsy from International Classification of Sleep Disorders, 2nd edition are as follows: ${ }^{1}$

\section{Narcolepsy with Cataplexy}

1. EDS almost daily for at least 3 months.

2. Definite history of cataplexy.

3. Diagnosis should be confirmed by one of the following:

a. PSG and MSLT; mean latency $\leq 8$ minutes and $\geq 2$ SOREMPS.

b. Orexin levels in the cerebrospinal fluid $110 \mathrm{pg} / \mathrm{mL}$ or one-third of mean normal controls.

4. Hypersomnia is not better explained by another disease or medication.

\section{Narcolepsy without Cataplexy}

1. EDS almost daily for at least 3 months.

2. Typical cataplexy is not present.

3. Diagnosis should be confirmed by PSG and MSLT; mean latency $\leq 8$ minutes and $\geq 2$ SOREMPs.

4. Hypersomnia is not better explained by another disorder or medication.

\section{Treatment}

The first line drug is modafinil which promotes wakefulness by interfering with the dopamine reuptake transporter. ${ }^{2}$ Tricyclic antidepressants and selective serotonin reuptake inhibitors reduce the severity and frequency of attacks in $98 \%$ of cataplexy patients. ${ }^{2}$

\section{Complications}

Falls, aspiration pneumonia, trauma, motor vehicle accidents, and depression are some of the complications.

\section{Case Discussion}

This particular patient did not have cataplexy which makes the diagnosis of narcolepsy tricky because none of the other symptoms are specific for narcolepsy. Also, narcolepsy is uncommon in the 6th decade of life. Presence of hypnagogic hallucinations led to suspicion of narcolepsy. It is not a common practice to look for additional symptoms in a subject presenting with EDS and possibility of narcolepsy is not considered unless PSG does not show OSA. Moreover, EDS covers wide range of diseases other than OSA. This case report highlights the need to look for such additional symptoms while evaluating a subject with EDS so that, the additional investigations like MSLT can be properly planned and communicated to the individual.

\section{Conclusion}

Narcolepsy is not a common disease especially in later years of life. A thorough history looking for additional symptoms other than EDS and proper planning of investigations is necessary to arrive at proper diagnosis.

Conflict of Interest

None declared.

\section{References}

1 SuzukiS,UeharaT, Ohira Y,Ikusaka M. Narcolepsy with cataplexy in an elderly woman. J Gen Intern Med 2015;30(8):1222-1224

2 Reynolds CF III. Diagnostic and statistical manual of mental disorders. 5th ed. Washington, DC; 2013:372-378

3 Berry RB. Fundamentals of sleep medicine. Elsevier Saunders; 2012:451-470

4 de Lecea L, Kilduff TS, Peyron C, et al. The hypocretins: hypothalamus-specific peptides with neuroexcitatory activity. Proc Natl Acad Sci U S A 1998;95(1):322-327

5 Thannickal TC, Moore RY, Nienhuis R, et al. Reduced number of hypocretin neurons in human narcolepsy. Neuron 2000;27(3):469-474

6 Thannickal TC, Nienhuis R, Siegel JM. Localized loss of hypocretin (orexin) cells in narcolepsy without cataplexy. Sleep 2009;32(8):993-998

7 Mignot E, Lin X, Hesla PE, Dement WC, Guilleminault C, Grumet FC. A novel HLA DR17,DQ1 (DQA1-0102/DQB1-0602 positive) haplotype predisposing to narcolepsy in Caucasians. Sleep 1993;16(8):764-765 